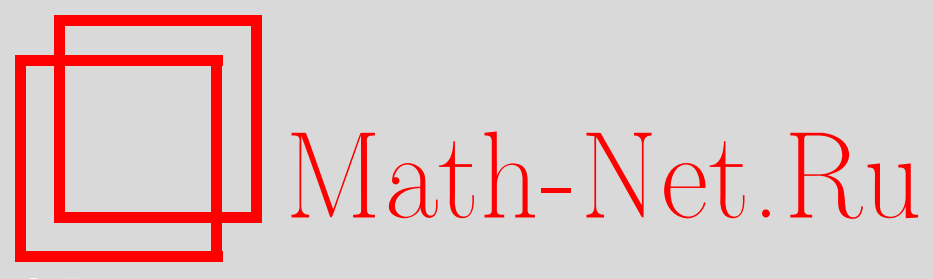

П. С. Дергач, Об однозначности алфавитного декодирования, Дискрет. матем., 2012, том 24, выпуск 4, 70-80

DOI: https://doi.org/10.4213/dm1211

Использование Общероссийского математического портала Math-Net.Ru подразумевает, что вы прочитали и согласны с пользовательским соглашением http://www. mathnet.ru/rus/agreement

Параметры загрузки:

IP : 52.90 .164 .192

26 апреля 2023 г., $10: 27: 27$ 


\title{
Об однозначности алфавитного декодирования
}

\author{
() 2012 г. П. С. Дергач
}

Целью этой работы является установление алгоритмической разрешимости проблемы однозначности алфавитного декодирования регулярных текстов.

\section{1. Введение}

А. А. Марковым было показано, что проблема однозначности алфавитного декодирования всех текстов над заданным конечным алфавитом $A$ сводится к декодированию конечного числа слов над алфавитом $A$ длины, не большей некоторой вычислимой величины, зависящей от длины схемы кодирования, мощности алфавита $A$ и других параметров [1]. В предлагаемой работе исследован случай, когда кодируемое множество слов является любым регулярным множеством. Показывается, что результат Маркова может быть обобщен на случай алфавитного декодирования любого регулярного множества слов над алфавитом $A$.

\section{2. Основные понятия и результаты}

Абстрактным конечным автоматом называется набор $V=(A, Q, B, \varphi, \psi)$, где $A, Q, B$ - конечные множества, $\varphi$ - функция, определенная на множестве $Q \times A$ и принимающая значения из $Q, \psi-$ функция, определенная на множестве $Q \times A$ и принимающая значения из $B$. Множества $A, Q, B$ называются, соответственно, входным алфавитом, алфавитом состояний и выходным алфавитом автомата $V$. Функция $\varphi$ называется функцией переходов, а функция $\psi-$ функцией выходов автомата $V$. Входными словами автомата $V=(A, Q, B, \varphi, \psi)$, называем произвольные конечные последовательности символов алфавита $A$. Для удобства рассматриваем при этом также пустое слово, не имеющее ни одного символа и обозначаемое $\Lambda$. Выходными словами алфавита $V$ называем конечные последовательности символов алфавита $B$, словами состояний - конечные последовательности символов алфавита $Q$ (в обоих случаях допускается и пустое слово $\Lambda$ ). Для каждого состояния автомата $V$ можно рассмотреть набор $(A, Q, B, \varphi, \psi, q)$, определяющий автомат $V$ с выделенным начальным состоянием $q$. Такие наборы $(A, Q, B, \varphi, \psi, q)$ называются инициальными абстрактными конечными автоматами; для них используется обозначение $V_{q}$.

Введем ряд понятий, связанных со словами. Пусть $C$ - некоторое конечное множество. Если $\gamma=c(1) \ldots c(n)-$ конечная последовательность символов $c(1), \ldots, c(n)$ алфавита $C$, то говорим, что $\gamma$ есть слово в алфавите $C$. Число $n$ называем длиной слова $\gamma$ и обозначаем через $|\gamma|$. Длина пустого слова равна 0 . Если $\gamma$ и $\delta-$ слова, причем $\gamma=\delta \delta^{\prime}$ 
для некоторого слова $\delta^{\prime}$, то говорим, что $\delta$ - начало слова $\gamma, \delta^{\prime}-$ конец слова $\gamma$. Множество всех слов в алфавите $C$ обозначаем $C^{*}$. Начало слова $\gamma$, имеющее длину $l$, обозначаем $\left[{ }_{l}(\gamma) \text {; окончание слова } \gamma \text {, имеющее длину } l \text {, обозначаем через }\right]_{l}(\gamma)$. Введем обозначения

$$
\left.\gamma_{l, m}=\right]_{m-l}([m(\gamma)), \quad|\gamma| \geqslant m>l \geqslant 1 .
$$

Функции переходов и выходов автомата $V=(A, Q, B, \varphi, \psi)$ доопределим на множестве $Q \times A^{*}$ (сохраним за ними те же обозначения). Именно, полагаем по определению

$$
\varphi(q, \Lambda)=q, \quad \varphi(q, \alpha a)=\varphi(\varphi(q, \alpha), a),
$$

где $q \in Q, \alpha \in A^{*}, a \in A$. Аналогично, полагаем

$$
\psi(q, \Lambda)=\Lambda, \quad \psi(q, \alpha a)=\psi(\varphi(q, \alpha), a) .
$$

Пусть $V_{q}=(A, Q, B, \varphi, \psi, q)$ - инициальный абстрактный конечный автомат, $B^{\prime} \subseteq B$. Множество

$$
M=\left\{\alpha \mid \alpha \in A^{*}, \psi(q, \alpha) \in B^{\prime}\right\}
$$

называем представимым в абстрактном конечном автомате $V_{q}$ с помощью подмножества $B^{\prime}$ выходных символов. Говорим также, что автомат $V_{q}$ представляет $M$ посредством $B^{\prime}$. Пусть $M \subseteq A^{*} \backslash\{\Lambda\}$. Если существует конечный автомат $V_{q}$, представляющий событие $M$ посредством некоторого подмножества $B^{\prime} \subseteq B$, то событие $M$ называем представимым.

Недетерминированным конечным автоматом называется набор $V=(A, Q, B, \gamma)$, где $A, Q, B$ - конечные множества, $\gamma-$ функция, определенная на множестве $Q \times A$ и принимающая в качестве своих значений подмножества множества $Q \times B$. Если для каждой пары $(q, a)$, где $q \in Q$ и $a \in A$ значение $\gamma(q, a)$ есть одноэлементное множество $\left(q^{\prime}, b\right)$, то можно определить функции $\varphi, \psi$ :

$$
\varphi(q, a)=q^{\prime}, \quad \psi(q, a)=b .
$$

При этом возникает некоторый абстрактный конечный автомат $V=(A, Q, B, \varphi, \psi)$. В указанном смысле абстрактный конечный автомат можно рассматривать как частный случай недетерминированного конечного автомата. Понятие инициального недетерминированного конечного автомата, аналогичное понятию инициального абстрактного конечного автомата, возникает, если выделено некоторое подмножество $Q^{\prime} \subseteq Q$ начальных состояний автомата. Пусть $Q^{\prime}$ - такое выделенное подмножество и $\alpha=a(1) \ldots a(s)-$ слово в алфавите $A$. Определим класс $\tilde{\gamma}\left(Q^{\prime}, \alpha\right)$ последовательностей $(q(1), b(1)), \ldots,(q(s), b(s))$. Каждая последовательность этого класса удовлетворяет следующим условиям:

(1) $q(1) \in Q^{\prime}$;

(2) $(q(i+1), b(i)) \in \gamma(q(i), a(i)), i=1, \ldots, s$ (в случае $i=s$ рассматривается значение $q(s+1)$, не включаемое в последовательность $\left.\tilde{\gamma}\left(Q^{\prime}, \alpha\right)\right)$.

Недетерминированный инициальный автомат $V=\left(A, Q, B, \gamma, Q^{\prime}\right)$ можно рассматривать как модель устройства, применяемого для распознавания входных последовательностей. Аналогично случаю обычного конечного автомата, при этом выделяется подмножество $B^{\prime}$ множества $B$ выходных символов. Множество входных слов $M$, распознаваемых автоматом, состоит из тех и только тех слов $\alpha \in A^{*}$, для которых в $\tilde{\gamma}\left(Q^{\prime}, \alpha\right)$ существует 
последовательность $(q(1), b(1)), \ldots,(q(s), b(s))$, удовлетворяющая условию $b(s) \in B^{\prime}$. Говорим также, что множество $M$ представимо в недетерминированном конечном автомате $V$ с помощью подмножества $B^{\prime}$ выходных символов.

Введем понятие обобщенного источника. Обобщенным источником в алфавите $A$ назовем конечный ориентированный граф $G$, у которого выделены начальная и финальная вершины $v, w, v \neq w$, причем каждому ребру приписано пустое слово $\Lambda$ либо символ алфавита $A$. Допускается наличие в графе петель и кратных ребер. Путем в обобщенном источнике $G$ будем называть последовательность

$$
\pi=\left(v_{1}, \rho_{1}, v_{2}, \rho_{2}, \ldots, \rho_{n}, v_{n+1}\right),
$$

где $v_{1}, v_{2}, \ldots, v_{n+1}$ - вершины графа $G, \rho_{i}$ - ребро графа $G$, ведущее от вершины $v_{i}$ к вершине $v_{i+1}, i=1, \ldots, n, n \geqslant 1$. Пути $\pi$ сопоставляем слово $[\pi]=a_{1} \ldots a_{n}$, где $a_{i}-$ символ алфавита $A$ либо пустое слово $\Lambda$, приписанное ребру $\rho_{i}, i=1, \ldots, n$. Говорим, что путь $\pi$ ведет от вершины $v_{1}$ к вершине $v_{n+1}$. Пусть $\alpha \in A^{*} \backslash\{\Lambda\}, u-$ вершина обобщенного источника $G$; множество всех вершин $u^{\prime}$ обобщенного источника $G$, для которых существует путь $\pi$, ведущий от $u$ к $u^{\prime}$ и такой, что $[\pi]=\alpha$, обозначим $\theta(u, \alpha)$. Каждый обобщенный источник $G$ с начальной вершиной $v$ и финальной вершиной $w$ определяет событие

$$
|G|=\left\{\alpha \mid \alpha \in A^{*} \backslash\{\Lambda\}, w \in \theta(v, \alpha)\right\} .
$$

Пусть $A=\left\{a_{1}, \ldots, a_{r}\right\}-$ произвольный конечный непустой алфавит. Пусть $P_{1}, P_{2}$ - непустые множества слов в алфавите $A$. Здесь и далее для удобства пустое слово за элемент множества $A^{*}$ не считается. Определим следующие операции над $P_{1}$ и $P_{2}$.

Произведение $P_{1} \cdot P_{2}$ множеств $P_{1}$ и $P_{2}$ есть множество всех слов вида $\alpha_{1} \alpha_{2}$, где $\alpha_{1} \in P_{1}, \alpha_{2} \in P_{2}$.

Итерация $\left(P_{1}\right)^{*}$ множества $P_{1}$ есть множество всех слов вида $\alpha_{1} \ldots \alpha_{k}$, где $\alpha_{1} \in P_{1}, \ldots$, $\alpha_{k} \in P_{1}, k \geqslant 1$.

Введем понятие регулярного множества в алфавите $A$. Множество $P \subseteq A^{*}$, называем регулярным в алфавите $A$, если его можно получить из множеств вида $\{a\}, a \in A$, применением конечного числа операций $\cup, \cdot,()^{*}$. Строгое определение регулярных множеств таково:

(1) $\{a\}$, где $a-$ произвольная буква алфавита $A$, есть регулярное множество в алфавите $A$;

(2) если $P_{1}, P_{2}$ - регулярные множества в алфавите $A$, то $P_{1} \cup P_{2}, P_{1} \cdot P_{2},\left(P_{1}\right)^{*}$ есть регулярные множества в алфавите $A$;

(3) регулярность произвольного множества в алфавите $A$ устанавливается в соответствии с (1)-(2) за конечное число шагов.

Введем понятие регулярного выражения в алфавите $A$. Регулярное выражение в алфавите $A$ представляет собой слово в алфавите $A \cup\{\vee, \cdot,(), *$,$\} , определяемое следующим$ образом:

(1) буквы алфавита $A$ - регулярные выражения в алфавите $A$;

(2) если $\alpha, \beta$ - регулярные выражения в алфавите $A$, то $(\alpha \vee \beta),(\alpha \cdot \beta),(\alpha)^{*}$ - регулярные выражения в алфавите $A$; 
(3) регулярность произвольного выражения в алфавите $A$ устанавливается в соответствии с (1)-(2) за конечное число шагов.

Сопоставим индуктивно каждому регулярному выражению $\mathfrak{P}$ в алфавите $A$ регулярное множество $|\mathfrak{\Re}|$ в алфавите $A$ следующим образом:

(1) множество $\{a\}$, если $\mathfrak{P}=a, a \in A$;

(2) множество $\left|\mathfrak{P}_{1}\right| \cup\left|\mathfrak{P}_{2}\right|$, если $\mathfrak{\Re}=\left(\mathfrak{P}_{1} \vee \mathfrak{P}_{2}\right)$;

(3) множество $\left|\mathfrak{P}_{1}\right| \cdot\left|\mathfrak{P}_{2}\right|$, если $\mathfrak{P}=\left(\mathfrak{P}_{1} \cdot \mathfrak{P}_{2}\right)$;

(4) множество $\left(\left|\mathfrak{P}_{1}\right|\right)^{*}$, если $\mathfrak{P}=\left(\mathfrak{P}_{1}\right)^{*}$.

Зафиксируем два конечных непустых алфавита $A$ и $B$.

Пусть дано некоторое отображение $f: A \rightarrow B^{*}$ :

$$
\begin{gathered}
f\left(a_{1}\right)=\beta_{1}, \\
f\left(a_{2}\right)=\beta_{2}, \\
\cdots \\
f\left(a_{r}\right)=\beta_{r} .
\end{gathered}
$$

Это соотношение называется схемой кодирования. Доопределим отображение $f$ до отображения $\tilde{f}: A^{*} \rightarrow B^{*}$ следующим образом:

$$
\tilde{f}\left(a_{i_{1}} a_{i_{2}} \ldots a_{i_{n}}\right)=\beta_{i_{1}} \beta_{i_{2}} \ldots \beta_{i_{n}} .
$$

Это отображение $\tilde{f}$ будем называть алфавитным кодированием.

Пусть есть некоторое регулярное множество $P$ в алфавите $A$ и некоторое алфавитное кодирование $f$. Пусть $\beta \in \tilde{f}(P)$. Тогда $\alpha \in P$ называется расшифровкой $\beta$ при алфавитном кодировании $\tilde{f}$ на регулярном множестве $P$ или расшифровкой $\beta$, если $f(\alpha)=\beta$. Таких расшифровок может быть несколько. Если для любых различных $\alpha_{1}, \alpha_{2} \in P$ верно, что $\tilde{f}\left(\alpha_{1}\right) \neq \tilde{f}\left(\alpha_{2}\right)$, то говорим, что декодирование однозначно на $P$ по $\tilde{f}$.

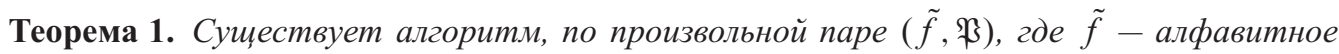
кодирование из алфавита $A$ в алфавит $B$, а $\mathfrak{P}$-регулярное выражение в алфавите $A$, определяюший, однозначно ли декодирование на $|\mathfrak{P}|$ по $\tilde{f}$.

\section{3. Вспомогательные утверждения}

Лемма 1. Если $R$ есть регулярное множество в алфавите A, представимое регулярным выражением $\mathfrak{P}$ в алфавите $A$ c $k$ операциями $\cdot, \vee$, то существует такой обобщенный источник $G$ в алфавите $A$, что $R=|G|$, причем в $G$ имеется не более, чем $4 k+2$ вериин.

Доказательство. Будем доказывать утверждение индукцией по числу операций в $\mathfrak{P}$. Если $R=\{a\}$, где $a \in A$, то искомый обобщенный источник $G$ указан на рис. 1 . Здесь через $v$ обозначена начальная вершина источника $G, w-$ финальная вершина источника $G$. Здесь $k=0$, и в $G$ две вершины. Для этого случая утверждение доказано.

Пусть

$$
\mathfrak{P}=\mathfrak{P}_{1} \vee \mathfrak{P}_{2} \text {, }
$$


$v$

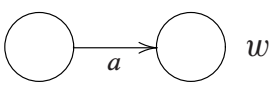

Рис. 1.

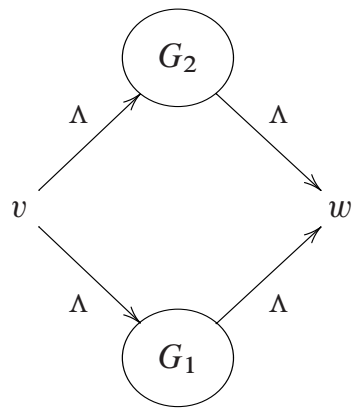

Рис. 2.

в $\mathfrak{P}_{1}$ содержится $k_{1}$ операций $\cdot, \vee$, в $\mathfrak{P}_{2}$ содержится $k_{2}$ операций $\cdot \vee$, и $G_{1}, G_{2}$ есть обобщенные источники в алфавите $A$, такие, что

$$
\left|\mathfrak{P}_{1}\right|=\left|G_{1}\right|, \quad\left|\mathfrak{P}_{2}\right|=\left|G_{2}\right| .
$$

По предположению индукции можно считать, что в $G_{1}$ содержится не более, чем $4 k_{1}+2$ вершин, а в $G_{2}$ содержится не более $4 k_{2}+2$ вершин. Можно считать, что множества вершин графов $G_{1}, G_{2}$ не пересекаются. Объединим графы $G_{1}, G_{2}$ и введем две новые вершины $v, w$. От $v$ проведем ребра с отметкой $\Lambda$ к начальным вершинам обобщенных источников $G_{1}, G_{2}$; к $w$ проведем ребра с отметкой $\Lambda$ от финальных вершин этих обобщенных источников. Получим обобщенный источник $G$ с начальной вершиной $v$ и финальной вершиной $w$, для которого

$$
|G|=\left|\mathfrak{P}_{1}\right| \vee\left|\mathfrak{P}_{2}\right|
$$

(см. рис. 2). В обобщенном источнике $G$ имеется не более

$$
\left(4 k_{1}+2\right)+\left(4 k_{2}+2\right)+2=4\left(k_{1}+k_{2}+1\right)+2
$$

вершин, а в $\mathfrak{P}$ имеется не более $k_{1}+k_{2}+1$ операций $\cdot, \vee$

Пусть

$$
\mathfrak{P}=\mathfrak{P}_{1} \cdot \mathfrak{P}_{2},
$$

в $\mathfrak{P}_{1}$ имеется $k_{1}$ операций $\cdot \vee$, в $\mathfrak{P}_{2}$ имеется $k_{2}$ операций $\cdot, \vee$, и пусть $G_{1}, G_{2}-$ обобщенные источники в алфавите $A$ такие, что

$$
\left|\mathfrak{P}_{1}\right|=\left|G_{1}\right|, \quad\left|\mathfrak{P}_{2}\right|=\left|G_{2}\right| .
$$

По предположению индукции можно считать, что в $G_{1}$ содержится не более, чем $4 k_{1}+2$ вершин, а в $G_{2}-$ не более $4 k_{2}+2$ вершин. Можно считать, что множества вершин графов 


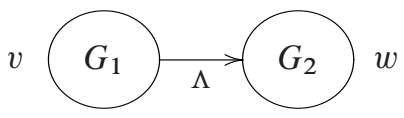

Рис. 3.

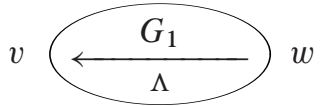

Pис. 4.

$G_{1}, G_{2}$ не пересекаются. Проведем от финальной вершины обобщенного источника $G_{1}$ ребро с отметкой $\Lambda$ к начальной вершине обобщенного источника $G_{2}$. Получим обобщенный источник $G$, начальной вершиной которого служит начальная вершина $v$ графа $G_{1}$, а финальной - финальная вершина $w$ графа $G_{2}$ (см. рис. 3 ). При этом

$$
|G|=\left|\mathfrak{P}_{1}\right| \cdot\left|\mathfrak{P}_{2}\right| \text {. }
$$

В обобщенном источнике $G$ имеется не более

$$
\left(4 k_{1}+2\right)+\left(4 k_{2}+2\right)+2=4\left(k_{1}+k_{2}+1\right)+2
$$

вершин, а в $\mathfrak{P}-$ не более $k_{1}+k_{2}+1$ операций $\cdot, \vee$

Пусть

$$
\mathfrak{P}=\left(\mathfrak{P}_{1}\right)^{*},
$$

в $\mathfrak{P}_{1} k_{1}$ операций и $G_{1}$ - обобщенный источник такой, что

$$
\left|\mathfrak{P}_{1}\right|=\left|G_{1}\right| \text {. }
$$

По предположению индукции можно считать, что в $G_{1}$ содержится не более, чем $4 k_{1}+2$ вершин. Проведем от финальной вершины $w$ обобщенного источника $G_{1}$ ребро с отметкой $\Lambda$ к начальной вершине $v$ этого обобщенного источника. Получим обобщенный источник $G$ с теми же начальной и финальной вершинами, что и $G_{1}$, причем

$$
|G|=\left|\left(\mathfrak{P}_{1}\right)^{*}\right|
$$

(см. рис. 4). В обобщенном источнике $G$ имеется не более $4 k_{1}+2$ вершин, а в $\mathfrak{P}$ имеется не более $k_{1}$ операций $\cdot, \vee$.

Таким образом, утверждение индукции, а с ним и лемма 1 , доказаны.

Лемма 2. Пусть $G$ - обобщеннный источник с $k$ вершинами. Тогда событие $|G|$ представимо автоматом с входным алфавитом $A$, выходным алфавитом $\{0,1\}$ и алфавитом состояний мощности $2^{k}$.

Доказательство леммы приведено в [2].

Лемма 3. Любой недетерминированный конечный автомат $V$ c q состояниями может быть преобразован в абстрактный конечный автомат $V^{\prime}$ с не более чем $2^{q}$ состояниями так, чтобы представляемые этими автоматами по $\{1\}$ множества совпадали. 
Доказательство леммы приведено в [2].

Лемма 4. Пусть $A-$ конечный алфавит; $\delta_{1}, \delta_{2}, \xi_{1}, \xi_{2}, \beta-$ слова (возможно, пустые) 8 алфавите $A ; B_{1}, B_{2}$ - регулярные множества в алфавите $A$ и $\delta_{1} \beta \delta_{2} \in B_{1}$, $\xi_{1} \beta \xi_{2} \in B_{2}$, u nycmb

$$
\begin{aligned}
& V_{1}=\left(A, Q_{1},\{0,1\}, \varphi_{1}, \psi_{1}, q_{1}\right), \\
& V_{2}=\left(A, Q_{2},\{0,1\}, \varphi_{2}, \psi_{2}, q_{2}\right)
\end{aligned}
$$

- инициальные абстрактные конечные автоматы, представляющие по множеству $\{1\}$ множества $B_{1}, B_{2}$ соответственно; тогда существует слово $\beta^{\prime}$ в алфавите $A$, $\left|\beta^{\prime}\right| \leqslant\left|Q_{1}\right|\left|Q_{2}\right|$, такое, что $\delta_{1} \beta^{\prime} \delta_{2} \in B_{1}, \xi_{1} \beta^{\prime} \xi_{2} \in B_{2}$.

Доказательство. Допустим, что $|\beta|>\left|Q_{1}\right|\left|Q_{2}\right|$. Рассмотрим множество $T$ пар состояний автоматов $V_{1}, V_{2}$ такое, что

$$
T=\left\{\left(\varphi_{1}\left(q_{1}, \delta_{1}\left[{ }_{l}(\beta)\right), \varphi_{2}\left(q_{2}, \xi_{1}\left[{ }_{l}(\beta)\right)\right), 1 \leqslant l \leqslant|\beta|\right\} .\right.\right.
$$

Так как

$$
|T|<\left|Q_{1}\right|\left|Q_{2}\right|+1,
$$

существуют $1 \leqslant l_{1}<l_{2} \leqslant\left|Q_{1}\right|\left|Q_{2}\right|+1$ такие, что

$$
\begin{aligned}
& \varphi_{1}\left(q_{1}, \delta_{1}\left[l_{1}(\beta)\right)=\varphi_{1}\left(q_{1}, \delta_{1}\left[l_{2}(\beta)\right),\right.\right. \\
& \varphi_{2}\left(q_{2}, \xi_{1}\left[l_{1}(\beta)\right)=\varphi_{2}\left(q_{2}, \xi_{1}\left[l_{2}(\beta)\right) .\right.\right.
\end{aligned}
$$

Пусть $\beta_{1}$ и $\beta_{2}$ удовлетворяют соотношениям

$$
\beta_{1}=\left[l_{1}(\beta), \beta=\left[l_{2}(\beta) \beta_{2} .\right.\right.
$$

Тогда

$$
\begin{aligned}
\psi_{1}\left(q_{1}, \alpha_{1} \beta_{1} \beta_{2} \alpha_{2}\right) & =\psi_{1}\left(\varphi_{1}\left(q_{1}, \alpha_{1} \beta_{1}\right), \beta_{2} \alpha_{2}\right) \\
& =\psi_{1}\left(\varphi_{1}\left(q_{1}, \alpha_{1}\left[l_{2}(\beta)\right), \beta_{2} \alpha_{2}\right)\right. \\
& =\psi_{1}\left(\varphi_{1}\left(q_{1}, \delta_{1}\left[l_{2}(\beta) \beta_{2}\right), \delta_{2}\right)\right. \\
& =\psi_{1}\left(\varphi_{1}\left(q_{1}, \delta_{1} \beta\right), \delta_{2}\right) \\
& =\psi_{1}\left(q_{1}, \delta_{1} \beta \delta_{2}\right)=1
\end{aligned}
$$

Аналогично получаем, что

$$
\psi_{2}\left(q_{2}, \xi_{1} \beta_{1} \beta_{2} \xi_{2}\right)=1 .
$$

Таким образом, существует $\beta^{\prime \prime}=\beta_{1} \beta_{2}$ такое, что $\delta_{1} \beta^{\prime \prime} \delta_{2} \in B_{1}, \xi_{1} \beta^{\prime \prime} \xi_{2} \in B_{2}$, и $\left|\beta^{\prime \prime}\right|<|\beta|$. Проведя конечное число раз процедуру сокращения $\beta$, получим $\beta^{\prime}$ длины, не большей $\left|Q_{1}\right|\left|Q_{2}\right|$, такое, что $\delta_{1} \beta^{\prime} \delta_{2} \in B_{1}, \xi_{1} \beta^{\prime} \xi_{2} \in B_{2}$. Лемма 4 доказана.

Лемма 5. Пусть $\alpha$ есть слово в алфавите $A$, и $B$ есть регулярное множество в алфавите $A$, представимое с помощью инициального абстрактного конечного автомата $V_{q_{0}}=\left(A, Q,\{0,1\}, \varphi, \psi, q_{0}\right)$ с помощью множества $\{1\}, \alpha \in B, 1 \leqslant s<|\alpha| ;$ тогда существуют регулярные множества $B_{1}$ и $B_{2}$ в алфавите $A$, представимые с помощью инициальных абстрактных конечных автоматов с входным алфавитом А, выходным алфавитом $\{0,1\}$ и алфавитом состояний $|Q|$ с помощью множества $\{1\}$ такие, что $\left.B_{1} \cdot B_{2} \subseteq B, \alpha \in B_{1} \cdot B_{2},{ }_{s}(\alpha) \in B_{1},\right]_{|\alpha|-s}(\alpha) \in B_{2}$. 
Доказательство. По леммам 1 и 2, существует инициальный абстрактный конечный автомат $V=\left(A, Q,\{0,1\}, \varphi, \psi, q_{0}\right)$, представляющий регулярное множество $B$ с помощью множества $\{1\}$. Пусть $\varphi\left(q_{0},{ }_{s}(\alpha)\right)=q_{1}$. Рассмотрим два инициальных абстрактных конечных автомата

$$
V_{1}=\left(A, Q,\{0,1\}, \varphi, \psi_{1}, q_{0}\right), \quad V_{2}=\left(A, Q,\{0,1\}, \varphi, \psi, q_{1}\right),
$$

где $\psi_{1}(q, a)=1$ при $\varphi(q, a)=q_{1}$ и $\psi_{1}(q, a)=0$ в противном случае. По теореме Клини (см. [2]), эти два автомата по множеству $\{1\}$ представляют регулярные множества $B_{1}$ и $B_{2}$. Так как $\varphi\left(q_{0},{ }_{s}(\alpha)\right)=q_{1}$, верно, что $\psi_{1}\left(q_{0},{ }_{s}(\alpha)\right)=1$, то есть ${ }_{s}(\alpha) \in B_{1}$. Далее, так как $\alpha \in B$,

$$
\begin{aligned}
1=\psi\left(q_{0}, \alpha\right) & =\psi\left(\varphi\left(q_{0},\left[{ }_{s}(\alpha)\right),\right]_{|\alpha|-s}(\alpha)\right) \\
& \left.\left.=\psi\left(q_{1},\right]_{|\alpha|-s}(\alpha)\right),\right]_{|\alpha|-s}(\alpha) \in B_{2}
\end{aligned}
$$

Значит, $\alpha \in B_{1} \cdot B_{2}$.

Пусть $\alpha_{1} \in B_{1}, \alpha_{2} \in B_{2}$. Тогда

$$
\varphi\left(q_{0}, \alpha_{1}\right)=q_{1}, \quad \psi\left(q_{1}, \alpha_{2}\right)=1
$$

Значит,

$$
\psi\left(q_{0}, \alpha_{1} \alpha_{2}\right)=\psi\left(\varphi\left(q_{0}, \alpha_{1}\right), \alpha_{2}\right)=\psi\left(q_{1}, \alpha_{2}\right)=1,
$$

то есть, $\alpha_{1} \alpha_{2} \in B$. Поэтому $B_{1} \cdot B_{2} \subseteq B$. Лемма 5 доказана.

Лемма 6. Пусть $\tilde{f}$ - алфавитное кодирование, $P$ - регулярное множество в алфавите $A=\left\{a_{1}, \ldots, a_{r}\right\}$, соответствующее регулярному выражению $\mathfrak{P}$ в алфавите $A$ c $k$ операииями $, \vee, \alpha_{1}, \alpha_{2} \in P, \alpha_{1} \neq \alpha_{2}, u \tilde{f}\left(\alpha_{1}\right)=\tilde{f}\left(\alpha_{2}\right)$. Тогда существуют такие $\alpha_{1}^{\prime}, \alpha_{2}^{\prime} \in P$, $\alpha_{1}^{\prime} \neq \alpha_{2}^{\prime}$, что $\tilde{f}\left(\alpha_{1}^{\prime}\right)=\tilde{f}\left(\alpha_{2}^{\prime}\right) u\left|\alpha_{1}^{\prime}\right|,\left|\alpha_{2}^{\prime}\right| \leqslant R_{f}^{2} 2^{2^{4 k+3} r R_{f}}$.

Доказательство. По лемме 1 , в алфавите $A$ существует обобщенный источник $G$ такой, что $P=|G|$ и в нем не более $4 k+2$ вершин. По лемме 2 , множество $|G|$ представимо абстрактным конечным автоматом с входным алфавитом $A$ и алфавитом состояний мощности не более $2^{4 k+2}$. Пусть $\alpha-$ некоторое слово в алфавите $A$ и $n \geqslant 2$. Пусть

$$
\Delta=[|\alpha| / n] .
$$

Тогда

$$
(\alpha)_{n, 1}=\left[{ }_{\Delta}(\alpha),(\alpha)_{n, 2}=\right]_{|\alpha|-\Delta}(\alpha) .
$$

Пусть $p=2 R_{f}$. Возможны два случая: во-первых,

$$
\left|\tilde{f}\left(\left(\alpha_{1}\right)_{p, 1}\right)\right| \leqslant\left|\tilde{f}\left(\left(\alpha_{2}\right)_{p, 1}\right)\right|
$$

и во-вторых,

$$
\left|\tilde{f}\left(\left(\alpha_{1}\right)_{p, 1}\right)\right|>\left|\tilde{f}\left(\left(\alpha_{2}\right)_{p, 1}\right)\right| .
$$

Разберем, например, первый случай. Второй случай разбирается аналогично. Заметим, что

$$
\begin{aligned}
& \left|\tilde{f}\left(\left(\alpha_{1}\right)_{p, 1}\right)\right| \leqslant R_{f}\left|\left(\alpha_{1}\right)_{p, 1}\right| \leqslant\left|\alpha_{1}\right| / 2 \leqslant\left|\tilde{f}\left(\alpha_{1}\right)\right| / 2, \\
& \left|\tilde{f}\left(\left(\alpha_{2}\right)_{p, 1}\right)\right| \leqslant R_{f}\left|\left(\alpha_{2}\right)_{p, 1}\right| \leqslant\left|\alpha_{2}\right| / 2 \leqslant\left|\tilde{f}\left(\alpha_{2}\right)\right| / 2
\end{aligned}
$$




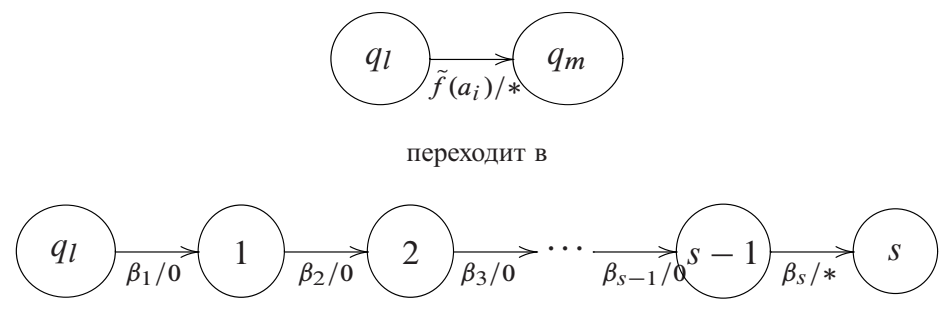

Рис. 5.

при этом,

$$
\left|\tilde{f}\left(\alpha_{1}\right)\right|=\left|\tilde{f}\left(\alpha_{2}\right)\right|
$$

Значит, части слова $\tilde{f}\left(\alpha_{1}\right)=\tilde{f}\left(\alpha_{2}\right)$, соответствующие словам $\tilde{f}\left(\left(\alpha_{1}\right)_{p, 1}\right)$ и $\tilde{f}\left(\left(\alpha_{2}\right)_{p, 1}\right)$, находятся в первой его половине. По лемме 5 , в алфавите $A$ существуют регулярные множества $A_{1,1}, A_{1,2}, A_{2,1}, A_{2,2}$ такие, что $\left(\alpha_{1}\right)_{p, i} \in A_{1, i},\left(\alpha_{2}\right)_{p, i} \in A_{2, i}, i=1,2, A_{1,1} \times A_{1,2} \subseteq P$, $A_{2,1} \times A_{2,2} \subseteq P$, и эти множества представимы, соответственно, абстрактными конечными автоматами $V_{1,1}, V_{1,2}, V_{2,1}, V_{2,2}$ с входным алфавитом $A$, выходным алфавитом $\{0,1\}$ и алфавитом состояний мощности не более $2^{4 k+2}$ с помощью множества $\{1\}$. Заменим в этих автоматах входной алфавит $A$ на $\tilde{f}(A)$ и соответственно поправим функции перехода и выхода, заменив в них $a_{i}$ на $\tilde{f}\left(a_{i}\right)$. Выходной алфавит, алфавит состояний и начальное состояние оставим теми же. Тогда регулярные множества $\tilde{f}\left(A_{1,1}\right), \tilde{f}\left(A_{1,2}\right)$, $\tilde{f}\left(A_{2,1}\right), \tilde{f}\left(A_{2,2}\right)$ представимы этими автоматами. Мощность их алфавитов состояний попрежнему не превосходит $2^{4 k+2}$. Теперь заменим для каждой пары состояний $q_{l}, q_{m}$ и каждого слова $\tilde{f}\left(a_{i}\right)$ имеющиеся в автоматах переходы из состояния $q_{l}$ в состояние $q_{m}$ по слову $\tilde{f}\left(a_{i}\right)=\beta_{1} \ldots \beta_{s}$ на последовательность состояний с побуквенными переходами $\beta_{1}, \ldots, \beta_{s}$ так, как это изображено на рис. 5 .

Таким образом, мы получаем недетерминированные конечные автоматы $\tilde{V}_{1,1}, \widetilde{V}_{1,2}$, $\tilde{V}_{2,1}, \widetilde{V}_{2,2}$, представляющие по $\{1\}$ множества $\tilde{f}\left(A_{1,1}\right), \tilde{f}\left(A_{1,2}\right), \tilde{f}\left(A_{2,1}\right), \tilde{f}\left(A_{2,2}\right)$ соответственно. У этих автоматов входным алфавитом, в отличие от автоматов $V_{1,1}, V_{1,2}, V_{2,1}$, $V_{2,2}$, является множество $B$. Оценим сверху мощность $S$ множества состояний автомата $V_{1,1}$ :

$$
S \leqslant 2^{4 k+2}+2^{4 k+2} r\left(R_{f}-1\right) \leqslant 2^{4 k+2} r R_{f}-1 .
$$

Такая же оценка верна и для мощности множеств состояний автоматов $\widetilde{V}_{1,2}, \widetilde{V}_{2,1}, \widetilde{V}_{2,2}$. По лемме 3 , существуют абстрактные конечные автоматы $\tilde{V}_{1,1}^{B}, \widetilde{V}_{1,2}^{B}, \tilde{V}_{2,1}^{B}, \widetilde{V}_{2,2}^{B}$, представляющие по $\{1\}$ множества $\tilde{f}\left(A_{1,1}\right), \tilde{f}\left(A_{1,2}\right), \tilde{f}\left(A_{2,1}\right), \tilde{f}\left(A_{2,2}\right)$ соответственно. При этом у каждого из этих автоматов не более чем $2^{2^{4 k+2} r R_{f}-1}$ состояний.

Теперь мы можем применить лемму 4 к паре регулярных множеств $\tilde{f}\left(A_{1,1}\right), \tilde{f}\left(A_{2,1}\right)$ и к словам $\tilde{f}\left(\left(\alpha_{1}\right)_{p, 1}\right), \tilde{f}\left(\left(\alpha_{2}\right)_{p, 1}\right)$, где

$$
\beta=\tilde{f}\left(\left(\alpha_{1}\right)_{p, 1}\right), \quad \delta_{1}=\delta_{2}=\gamma_{1}=\Lambda, \quad \gamma_{2}=\tilde{f}\left(\alpha_{2}\right)_{\left|\tilde{f}\left(\left(\alpha_{1}\right)_{p, 1}\right)\right|,\left|\tilde{f}\left(\left(\alpha_{2}\right)_{p, 1}\right)\right|} .
$$

Из нее следует, что существует слово $\beta^{\prime} \in B^{*},\left|\beta^{\prime}\right| \leqslant 2^{2^{4 k+3} r R_{f}-2}$, такое, что $\beta^{\prime} \in \tilde{f}\left(A_{1,1}\right)$, $\beta^{\prime} \gamma_{2} \in \tilde{f}\left(A_{2,1}\right)$. Поэтому существуют слова $\alpha^{\prime} \in A_{1,1}, \alpha^{\prime \prime} \in A_{2,1}$ такие, что $\tilde{f}\left(\alpha^{\prime}\right)=\beta^{\prime}$, 
$\tilde{f}\left(\alpha^{\prime \prime}\right)=\beta^{\prime} \gamma_{2}$. Обозначим через $\lambda_{1}$ слово $\alpha^{\prime}\left(\alpha_{1}\right)_{p, 2} \in A_{1,1} \times A_{1,2} \subseteq P$ и через $\lambda_{2}$ слово $\alpha^{\prime \prime}\left(\alpha_{2}\right)_{p, 2} \in A_{2,1} \times A_{2,2} \subseteq P$. Тогда

$$
\begin{aligned}
& \tilde{f}\left(\lambda_{1}\right)=\tilde{f}\left(\alpha^{\prime}\left(\alpha_{1}\right)_{p, 2}\right)=\tilde{f}\left(\alpha^{\prime}\right) \tilde{f}\left(\left(\alpha_{1}\right)_{p, 2}\right)=\beta^{\prime} \tilde{f}\left(\left(\alpha_{1}\right)_{p, 2}\right), \\
& \tilde{f}\left(\lambda_{2}\right)=\tilde{f}\left(\alpha^{\prime \prime}\left(\alpha_{2}\right)_{p, 2}\right)=\tilde{f}\left(\alpha^{\prime \prime}\right) \tilde{f}\left(\left(\alpha_{2}\right)_{p, 2}\right)=\beta^{\prime} \gamma_{2} \tilde{f}\left(\left(\alpha_{2}\right)_{p, 2}\right) .
\end{aligned}
$$

Итак,

$$
\tilde{f}\left(\lambda_{1}\right)=\tilde{f}\left(\lambda_{2}\right)
$$

Пусть различие в расшифровках $\alpha_{1}, \alpha_{2}$ слова $\tilde{f}\left(\alpha_{1}\right)=\tilde{f}\left(\alpha_{2}\right)$ есть в его второй половине. Тогда и у $\tilde{f}\left(\lambda_{1}\right)=\tilde{f}\left(\lambda_{2}\right)$ есть различные расшифровки $\tilde{f}\left(\lambda_{1}\right), \tilde{f}\left(\lambda_{2}\right)$, так как во второй половине слова расшифровки не изменились. Если же это не так, то различие в расшифровках $\alpha_{1}, \alpha_{2}$ слова $\tilde{f}\left(\alpha_{1}\right)=\tilde{f}\left(\alpha_{2}\right)$ есть в его первой половине. В этом случае такие же рассуждения применяются к записанному в обратном порядке слову $\tilde{f}\left(\alpha_{1}\right)=\tilde{f}\left(\alpha_{2}\right)$.

Итак, можно считать, что

$$
\left|\left(\alpha_{1}\right)_{p, 1}\right| \leqslant 2^{2^{4 k+3} r R_{f}-2} .
$$

Ho

$$
\left|\left(\alpha_{1}\right)_{p, 1}\right| \geqslant\left|\alpha_{1}\right| / 2 R_{f}-1
$$

Поэтому

$$
\left|\alpha_{1}\right| \leqslant 2 R_{f}\left(\left|\left(\alpha_{1}\right)_{p, 1}\right|+1\right) \leqslant R_{f} 2^{2^{4 k+3} r R_{f}} .
$$

Отсюда следует, что

$$
\left|\alpha_{2}\right| \leqslant\left|\tilde{f}\left(\alpha_{2}\right)\right|=\left|\tilde{f}\left(\alpha_{1}\right)\right| \leqslant R_{f}\left|\alpha_{1}\right| \leqslant R_{f}^{2} 2^{2^{4 k+3} r R_{f}} .
$$

Утверждение леммы 6 доказано.

Следствие 1. Пусть $\tilde{f}$ - алфавитное кодирование, $P$ - регулярное множество в алфавите $A=\left\{a_{1}, \ldots, a_{r}\right\}$, представимое с помощью инициального абстрактного конечного автомата $V_{q_{0}}=\left(A, Q,\{0,1\}, \varphi, \psi, q_{0}\right)$ с помощью множества $\{1\}$. Тогда существуют такие $\alpha_{1}^{\prime}, \alpha_{2}^{\prime} \in P, \alpha_{1}^{\prime} \neq \alpha_{2}^{\prime}$, что $\tilde{f}\left(\alpha_{1}^{\prime}\right)=\tilde{f}\left(\alpha_{2}^{\prime}\right)$ u $\left|\alpha_{1}^{\prime}\right|,\left|\alpha_{2}^{\prime}\right| \leqslant R_{f}^{2} 4^{r R_{f}}|Q|$.

Доказательство. Доказательство полностью повторяет доказательство леммы 6. Искомую оценку получаем из цепочки неравенств

$$
\begin{aligned}
2 R_{f}\left(\left(2^{|Q|+r\left(R_{f}-1\right)|Q|}\right)^{2}+1\right) R_{f} & \leqslant 2 R_{f}^{2}\left(\left(2^{\left(r R_{f}|Q|-1\right)}\right)^{2}+1\right) \\
& =2 R_{f}^{2}\left(4^{r R_{f}|Q|-1}+1\right) \leqslant R_{f}^{2} 4^{r R_{f}|Q|} .
\end{aligned}
$$

Лемма 7. Сущзествует алгоритм, определяющий по произвольной паре $(\tilde{f}, \mathfrak{\Re})$, где $\tilde{f}-$ алфавитное кодирование из алфавита $A$ в алфавит $B$, а $\mathfrak{P}$-регулярное выражение $в$ алфавите $A$, существуют ли $\alpha_{1}, \alpha_{2} \in|\mathfrak{P}|, \alpha_{1} \neq \alpha_{2}$, такие, что выполняется равенство $\tilde{f}\left(\alpha_{1}\right)=\tilde{f}\left(\alpha_{2}\right)$. 
Доказательство. Проверим конечным перебором слов из $|\Re|$ длины, не превосходящей $R_{f}^{2} 2^{2^{4 k+3} r R_{f}}$, существуют ли два слова $\alpha_{1}, \alpha_{2} \in|\mathfrak{R}|, \alpha_{1} \neq \alpha_{2}$, такие, что $\tilde{f}\left(\alpha_{1}\right)=\tilde{f}\left(\alpha_{2}\right)$ и $\left|\alpha_{1}\right|,\left|\alpha_{2}\right| \leqslant R_{f}^{2} 2^{2^{4 k+3} r R_{f}}$. Если такие слова есть, то $\tilde{f}$ не является однозначно декодируемым на $|\mathfrak{\beta}|$. Если таких слов нет, то, по лемме $6, \tilde{f}$ однозначно декодируемо на $|\mathfrak{P}|$. Алгоритм проверки однозначности алфавитного декодирования, определяющий по произвольной паре $(\tilde{f}, \mathfrak{\beta})$, однозначно ли декодируемо $\tilde{f}$ на $|\mathfrak{\Re}|$, построен. Утверждение леммы 7 доказано.

Нетрудно видеть, что из леммы 7 вытекает утверждение теоремы 1.

\section{Список литературы}

1. Яблонский С. В., Введение в дискретную математику. Наука, Москва, 1986.

2. Кудрявцев В. Б., Алешин С. В., Подколзин А. С., Введение в теорию автоматов. Наука, Москва, 1985.

Статья поступила 16.03.2012. 